\title{
Defect modes of a two-dimensional photonic crystal in an optically thin dielectric slab
}

\author{
O. Painter, J. Vučković, and A. Scherer \\ California Institute of Technology, Electrical Engineering, MS 136-93, Pasadena, California 91125
}

Received June 30, 1998; revised manuscript received October 19, 1998

\begin{abstract}
We present a three-dimensional finite-difference time-domain analysis of localized defect modes in an optically thin dielectric slab that is patterned with a two-dimensional array of air holes. The symmetry, quality factor, and radiation pattern of the defect modes and their dependence on the slab thickness are investigated. (C) 1999 Optical Society of America [S0740-3224(99)01302-8]

OCIS codes: $250.0250,140.5960$.
\end{abstract}

\section{INTRODUCTION}

As early as 1946 Edward Purcell ${ }^{1}$ described the modification of the coupling of matter to the electromagnetic field by placing the system in a cavity. Almost forty years later Kleppner ${ }^{2}$ reinvestigated the idea of cavity modification of spontaneous emission, and later Yablonovitch ${ }^{3}$ applied these concepts to the modification of spontaneous emission in photonic bandgap materials. ${ }^{4}$ The use of electromagnetic cavities to control spontaneous emission is now an active field of research. Owing to the inherent size scale, initial studies were focused on cavity structures for microwave devices. ${ }^{5,6}$ With the maturation of nanometer-size photonic crystal fabrication in semiconductors $^{7-9}$ and other dielectrics ${ }^{10,11}$ there has been strong interest in creating optical microcavities for spontaneous emission control.

The VCSEL was the first device to utilize an optical cavity to shrink the optical mode to sizes of the order of the wavelength of light while still maintaining the low modal loss required of a laser. ${ }^{12}$ The microdisk laser is another example of the successful implementation of a high quality factor $(Q)$, dielectric optical microcavity with a mode volume of the order of a cubic wavelength. ${ }^{13-15}$ More recently studies were done by several groups on high- $Q$, ultrasmall volume cavities in photonic wire/ waveguide structures with a one-dimensional in-plane photonic crystal. ${ }^{16-18}$ Two-dimensional (2D) photonic crystals have also been fabricated and characterized in a variety of semiconductor materials ${ }^{9,19-22}$; however they are not effective, by themselves, in confining optical modes in the third direction. Three-dimensional (3D) photonic crystals can be used to trap light in all three directions, and they, too, have been fabricated at optical wavelengths in semiconductors, ${ }^{8}$ although of lesser quality and with much more difficulty than in the $2 \mathrm{D}$ case.

Technologically, a more appealing method of localizing light in all directions is the combination of a microdisktype structure and a $2 \mathrm{D}$ photonic crystal. ${ }^{23}$ This structure, shown in Fig. 1, is composed of a thin dielectric slab, for total internal reflection of the light in the vertical direction, and a $2 \mathrm{D}$ photonic crystal to provide in-plane lo- calization. The optical cavity itself is created by a defect in the photonic crystal, thereby forming an optical mode localized to the defect region. ${ }^{24}$ Also, the $2 \mathrm{D}$ photonic crystal reduces spontaneous emission into many of the lateral radiation modes present in the microdisk. ${ }^{15} \mathrm{Un}$ like the microdisk the $2 \mathrm{D}$ patterned dielectric slab is connected laterally; thus the in-plane radiation from the defect mode can be coupled efficiently to a waveguide for inplane routing. This opens up the possibility of a lowthreshold, single-mode, in-plane analog to the VCSEL. Such an in-plane source might be a useful component in low-power, high-density optoelectronic systems. ${ }^{25}$ Single-mode, high- $Q$, small volume optical cavities also hold the promise of highly efficient, low-noise LED's. ${ }^{26}$

We begin in Section 2 by outlining the important characteristics of optical cavity modes in the design of semiconductor light emitters. In Section 3 we analyze the properties of a $2 \mathrm{D}$ photonic crystal in a high-index slab with thickness of the order of the wavelength of light. We then perform a 3-D finite-difference time-domain (FDTD) analysis ${ }^{27}$ of localized defect modes of the photonic crystal in Section 4 . In Section 5 we conclude with a study of the effect of a symmetry breaking defect, which splits the defect mode degeneracy.

\section{DISCUSSION OF PROBLEM}

A number of key parameters determine the performance of an optical cavity in a semiconductor light emitter. Here we limit our discussion to design issues relevant to low-power, low-noise devices, such as those described in the Section 1. The $\beta$ factor is one of the most important characteristics of the optical-cavity semiconductor system. For a given mode $m, \beta_{m}$ is the fraction of spontaneous emission that is channeled into $m$, as opposed to all the other modes that are supported by the cavity. An optical mode $m$ in a microcavity is also described by its quality factor $Q$. The $Q$ of a cavity mode is equal to the mode frequency divided by the modal loss rate $Q=\omega / \gamma$. The loss rate $\gamma$ is the number of photons lost from the optical mode per second. Here we consider lost photons to be 


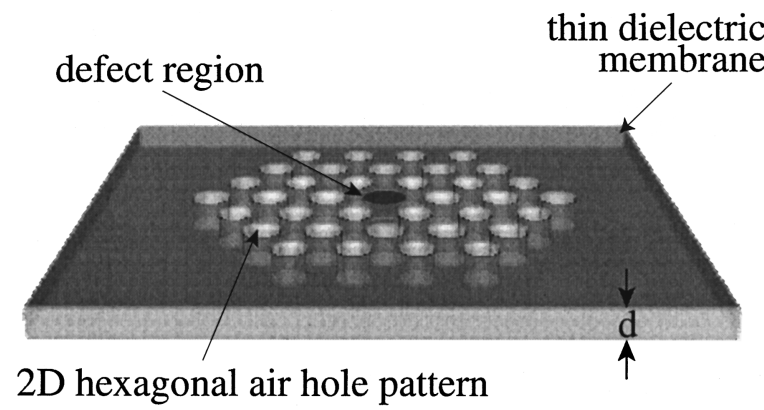

Fig. 1. Schematic of the 2D patterned dielectric slab. The slab is surrounded by air, and the $2 \mathrm{D}$ array of holes completely perforate the slab.

those that escape outside the cavity where they can be collected; in other words, we analyze a cold cavity in which gain and absorption are neglected.

For low-power semiconductor lasers, the figure of merit is the threshold current. A simple relation for the threshold current of a semiconductor laser can be written as follows ${ }^{28}$.

$$
I_{\mathrm{th}} \approx\left(\frac{q N_{o} V_{\mathrm{active}}}{\tau_{\mathrm{sp}}}+\frac{q \gamma}{\beta}\right)+I_{\mathrm{nr}} .
$$

The first term on the right-hand side of Eq. (1) represents the current needed to reach transparency in the semiconductor, defined by the transparency carrier density $N_{o}$. The transparency current is proportional to the total spontaneous emission rate into all optical modes $\tau_{\mathrm{sp}}^{-1}$ and to the volume of the active region $V_{\text {active }}$. The second term in Eq. (1) represents the current needed to overcome losses in the optical cavity. The final term $I_{\mathrm{nr}}$ is the current that is required for supplying all the nonradiative processes inside the semiconductor, such as leakage over the barriers of the quantum wells, Auger recombination, and surface recombination. $\quad \beta$ and $\tau_{\mathrm{sp}}$ are also important LED parameters. However, in an LED one is not concerned with reaching a lasing threshold; therefore it is beneficial in this case to lower $\tau_{\mathrm{sp}}$ to reduce the effects of nonradiative processes and to improve the modulation speed. $^{26}$

A careful analysis of spontaneous emission in semiconductor microcavities ${ }^{15,29}$ shows that the spontaneous emission rate into a single-cavity mode is inversely proportional to the optical mode volume. In a semiconductor the optical mode volume is in fact an effective mode volume that also contains information about the overlap of the active region and the optical mode. ${ }^{23}$ The challenge is to design and build a microcavity that has the optical properties of small volume, near unity $\beta$ factor, and large $Q$, while simultaneously the nonradiative processes are limited in the semiconductor.

Here we are interested in forming an optical cavity by using a $2 \mathrm{D}$ photonic crystal in an optically thin dielectric slab. A straightforward method of forming periodic dielectric structures is to etch holes in the material. The etched surface quality, and the resulting surface-state trap density, pose a large problem in many materials, such as GaAs. The InGaAsP-InP material system is a promising candidate for implementation of photonic- crystal-based light emitters. Because of the low surface recombination velocity ${ }^{23,30}$ in InGaAsP, one can reduce the nonradiative surface recombination loss by an order of magnitude or two relative to GaAs. For this reason we chose to design around this material system. Preliminary work on the fabrication of these microcavities in an InGaAsP multi-quantum-well structure is shown in Fig. 2(a), which shows a top view of the cavity, and in Fig. 2(b), which shows a cross section of the undercut patterned membrane. ${ }^{31}$ The quantum wells in this structure $^{32}$ were designed to have larger gain for electricfield components polarized in the plane of the quantum wells (TE gain). For this reason we focus here on designing an optical cavity that supports localized TE-like modes that will couple much more strongly to the electrons in the quantum well than TM-like modes.

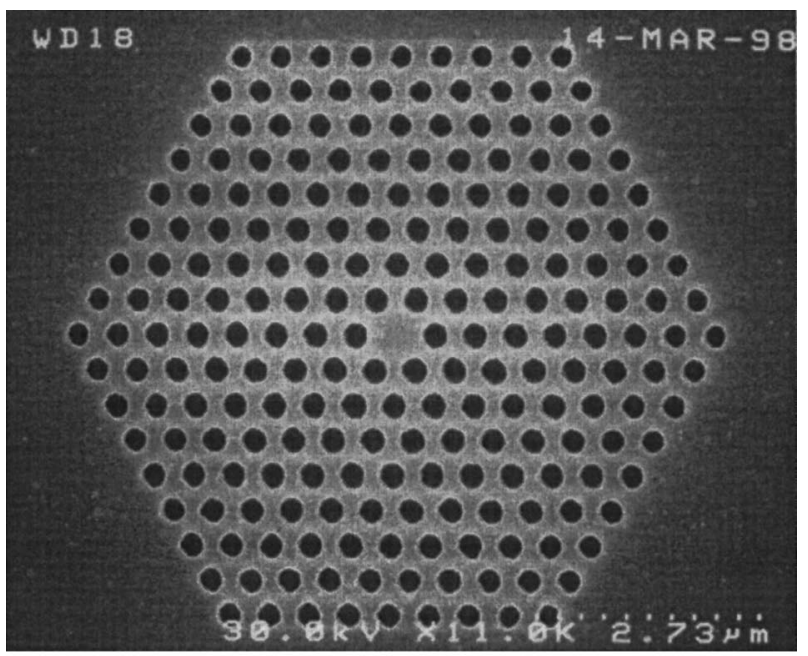

(a)

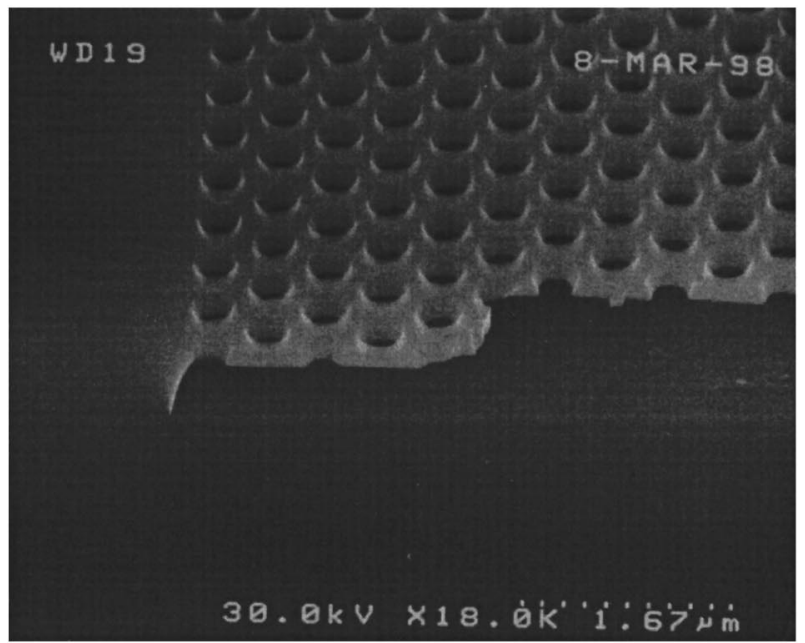

(b)

Fig. 2. (a) Top view of a microfabricated $2 \mathrm{D}$ hexagonal array of air holes with a single central hole missing. The interhole spacing a is $500 \mathrm{~nm}$, and the radius of the holes is approximately 150 nm. (b) Cross section through the patterned membrane structure. The thickness of the undercut membrane is $180 \mathrm{~nm}$, approximately one half-wavelength in the material. The membrane contains four strained quaternary quantum wells, optimized for $1.55-\mu \mathrm{m}$ emission wavelength. 


\section{TWO-DIMENSIONAL PHOTONIC CRYSTAL DESIGN}

In this section we analyze the $2 \mathrm{D}$ hexagonal pattern of air holes, without any defects, to establish the properties of the photonic crystal in a thin slab. We begin the analysis with a 2D-band-structure calculation in which the dielectric slab is infinitely thick. The effects of vertical guiding on the photonic crystal modes are approximated by replacement of the slab material refractive index with an effective refractive index ${ }^{30}$ of propagation for a guided slab mode. We then perform a 3D FDTD calculation to investigate more accurately the $2 \mathrm{D}$ band structure for a slab of finite thickness.

\section{A. Two-Dimensional Band Structure}

An illustration of the top view of the microcavity is shown in Fig. 3. The microcavity is composed of a dielectric material with a hexagonal array of air holes. In this subsection we treat the structure as infinite in the third direction. The modes of the structure can be classified as TE (electric field polarized normal to the axis of the infinitely long air holes) and TM (electric field polarized parallel to air hole axis). ${ }^{4}$ The properties of the $2 \mathrm{D}$ photonic crystal are determined by the interhole spacing $a$, the ratio of the radius of the air holes to the spacing $r / a$, and the refractive index of the material $n_{\text {slab }}$. The ratio $r / a$ affects the size of the TE and TM bandgaps. Also, as the $r / a$ ratio is increased, the frequencies of the photonic crystal modes tend to rise, owing to the larger air fraction and resulting lower average index. ${ }^{4}$ In the $2 \mathrm{D}$ calculations we take $r / a$ equal to 0.3 , chosen conservatively to lessen the fabrication tolerances. The refractive index of InGaAsP is approximately 3.4 at a wavelength of $1.55 \mu \mathrm{m} .^{33}$ This gives an effective index of 2.65 for the fundamental TE guided mode in a half-wavelength-thick slab. We use this effective index as the refractive index of the slab to approximate the effects of waveguiding on the photonic crystal modes in a thin slab. This approximation dramatically reduces the computation time necessary for full $3 \mathrm{D}$ calculations and is generally quite useful as a guide in determining the fundamental bandgap frequency and lowerlying frequency band dispersion curves.

Dispersion diagrams showing normalized frequency versus in-plane wave vector for TE and TM modes of the 2D photonic crystal are given in Figs. 4 and 5, respectively. The band-structure calculation was performed with the plane-wave expansion method. ${ }^{34}$ The band diagrams show a frequency bandgap for TE-polarized modes but no gap for TM modes with this refractive-index contrast and relatively small $r / a$. This limits the number of possible localized defect modes below, because TM modes will in general not be well contained by the photonic crystal. Throughout the paper we use a normalized frequency $a / \lambda_{o}$ to describe the band frequencies in the photonic crystal, where $\lambda_{o}$ is the wavelength in air. In addition we use the term dielectric band to describe the lower-frequency band that defines the bandgap and air band to describe the top band. In the TE-band diagram of Fig. 4 the fundamental bandgap is centered near a normalized frequency of 0.3 and is formed between the air band at the $\mathrm{X}$ point and the dielectric band at the $\mathrm{J}$ point.

\section{B. Three-Dimensional Finite-Difference Time-Domain Analysis of the Two-Dimensional Patterned Slab Waveguide}

In Subsection 3.A the 2D photonic crystal was formed from infinitely long air holes in an infinitely thick dielectric slab. Here we shrink the dielectric slab to a thickness of the order of the wavelength of light and surround it with air, thus providing a high degree of localization in the third direction. The properties of the $2 \mathrm{D}$ photonic crystal are still determined by $a, r / a$, and $n_{\text {slab }}$, but now the thickness of the slab is also important.

The resonant modes (leaky and guided) of a symmetric dielectric slab waveguide separate out into TE and TM polarizations that also have even or odd spatial symmetry

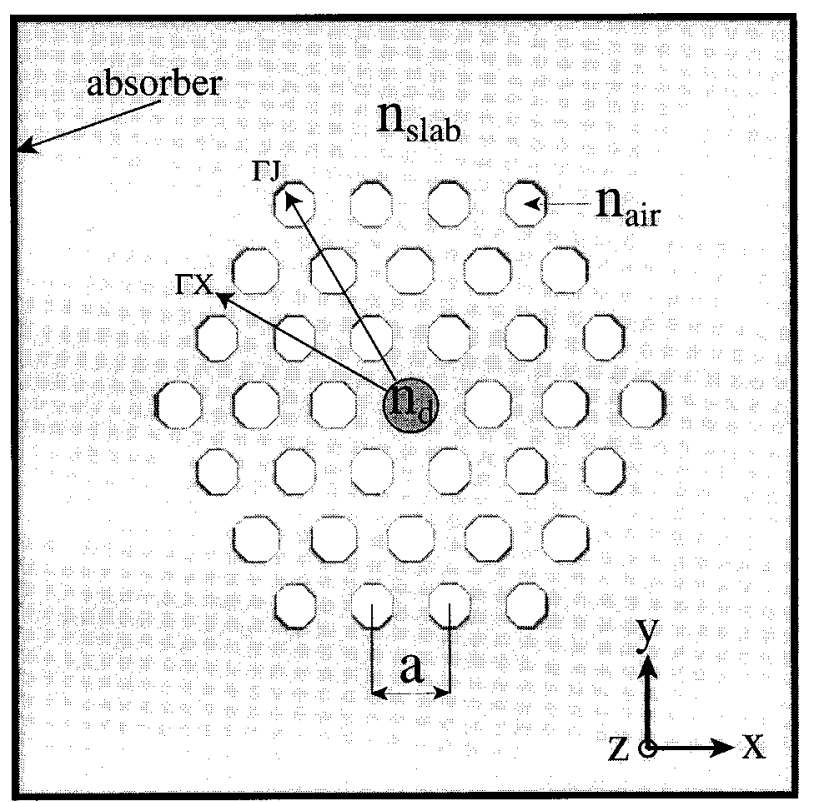

Fig. 3. Schematic of a 2D slice through the middle of the patterned high-index slab. The center hole has a refractive index larger than air, $n_{d}$, which forms a defect in the hexagonal lattice of air holes.

\section{TE Band Diagram}

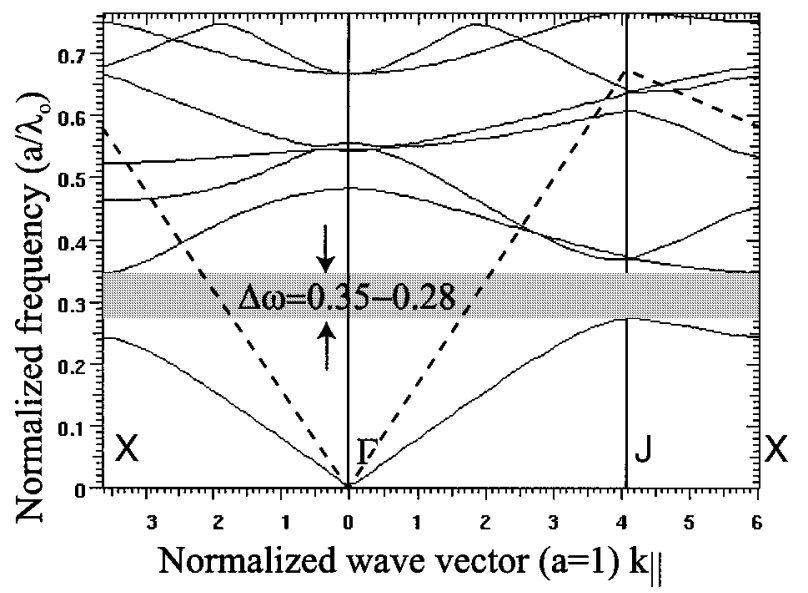

Fig. 4. Band diagram for TE-polarized light ( $E$ field polarized in plane). The holes have an index $n_{\text {air }}=1$. The material has an index $n_{\text {slab }}=2.65$. The radius of the holes is defined by the ratio $r / a=0.3$. The resulting TE bandgap extends between a normalized frequency, $\Delta \omega_{\text {gap }}=a / \lambda_{o}=0.28-0.35$. 


\section{TM Band Diagram}

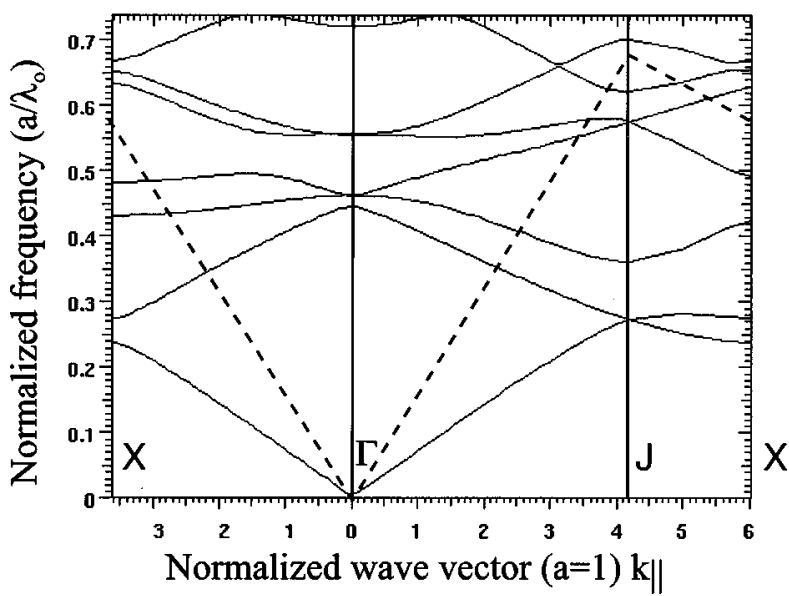

Fig. 5. Band diagram for TM-polarized light ( $E$ field polarized in the $\hat{z}$-direction). In this case the index contrast and $r / a$ are not large enough to open a full 2D bandgap between the first (dielectric) and second (air) bands.

with respect to the center of the waveguide. The TE modes have the electric field polarized in the plane of the waveguide, and the TM modes have the magnetic field polarized in the plane of the waveguide. The resonant modes of a 2D patterned dielectric slab surrounded by air, however, are not purely TE or TM but rather what we designate TE-like and TM-like. The TE-like and TM-like modes are classified by how they transform under the horizontal mirror operation in the middle of the dielectric slab. TE-like modes are even under reflection, and TMlike modes are odd. TE-like modes are composed of even TE slab modes and odd TM slab modes, whereas TM-like modes are formed from even TM slab modes and odd TE slab modes. As mentioned in Section 2, we are interested primarily in active regions with predominantly TE gain. Only the even TE slab modes will couple to such quantum wells placed at the center of the waveguide, or, in the 2D patterned waveguides, coupling will be limited to the TElike modes. For this reason we focus on designing defect cavities that support TE-like localized modes. Also, depending on the thickness of the dielectric slab, there can be higher-order guided modes supported by the patterned slab. $^{35}$ Here we consider dielectric slabs approximately a half-wavelength thick in which there is a bandgap between the fundamental (0th order) air and dielectric band TE-like modes.

A 3D FDTD simulation is used to model the fundamental TE-like band structure of the optically thin patterned waveguide. By applying appropriate Bloch boundary conditions over a unit cell of the photonic crystal, one can obtain the spectral response for a given in-plane wave vector. The peaks in the frequency spectrum give the eigenmodes of the photonic crystal at the $k$ vector determined by the boundary conditions. The interested reader is referred to the paper by Chan et al..$^{36}$ for further details. In our case the unit cell consists of an in-plane geometry given by the $2 \mathrm{D}$ unit cell of the hexagonal lattice. In the $\hat{z}$ direction we do not have periodicity, so a full description of the slab and surrounding air must be given. The Bloch boundary condition is applied to all four sides normal to plane of the slab, and Mur's absorbing boundary condition ${ }^{37}$ is applied to the top boundary. At the bottom boundary we apply an even mirror reflection positioned at the middle of the slab to select out only the TE-like modes of the structure. A uniform spatial resolution of 20 points per interhole spacing is used to discretize the unit cell, and the initial field is evolved for $2^{14}$ time steps. This gives a normalized frequency resolution of 0.0012 and a spatial resolution of approximately 20 points per wavelength in the high-index slab for frequencies within the bandgap. In these $3 \mathrm{D}$ calculations the real slab refractive index of 3.4 is used as opposed to the effective index from Subsection 3.A.

The TE-like band structure for a $2 \mathrm{D}$ patterned slab waveguide with thickness $d=0.4 a$ is plotted in Fig. 6 . The light line, indicated by a solid line in Fig. 6 and a dashed line in Fig. 4, is the dividing line between guided and leaky modes of the perforated dielectric slab. The light line is simply the linear dispersion curve of a photon in air (as the thin slab is surrounded by air in this structure). The region above the light line corresponds to leaky modes in which the optical mode leaks energy into the surrounding air as it propagates down the waveguide. The parts of the frequency bands that are below the light line are guided and do not leak energy as they propagate. Only the regions of the frequency bands that are guided are displayed in Fig. 6. The air band is guided near the band edge at the $\mathrm{X}$ point but eventually becomes leaky as it moves toward the $\Gamma$ point. The dielectric band, however, is guided throughout $k$ space. In the approximate TE band structure of Fig. 4 the frequency bands are plotted throughout $k$ space, showing both the guided and the leaky regions. The approximate $2 \mathrm{D}$ model of Subsection 3.A and the 3D model presented here compare well for the first few lower-lying bands.

A plot of the air band edge at the $X$ point and the dielectric band edge at the $\mathrm{J}$ point for varying slab thicknesses is given in Fig. 7. Also shown in this figure is the midgap frequency. A plot of the bandgap width is given in Fig. 8. As expected the midgap frequency increases as

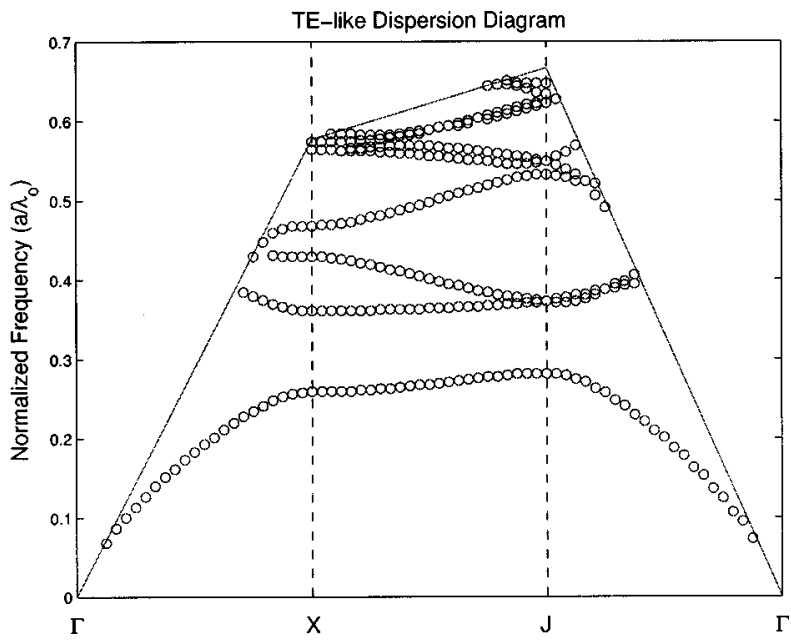

Fig. 6. Band structure of the TE-like modes of the 2D patterned slab waveguide surrounded by air $(d=0.4 a)$. The solid line represents the light line. Only the guided modes are plotted. 


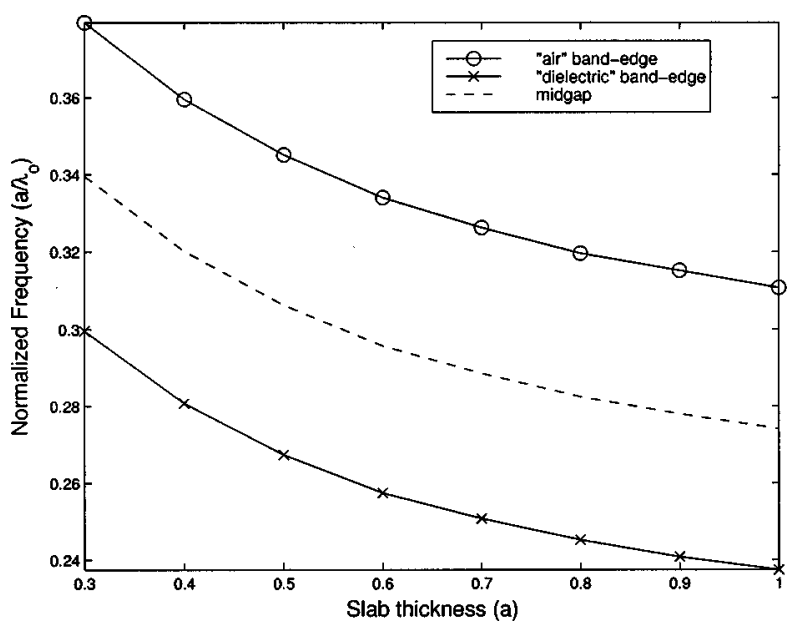

Fig. 7. Plot of the air and dielectric band edges as a function of slab thickness. The midgap frequency is also plotted, as a dashed curve.

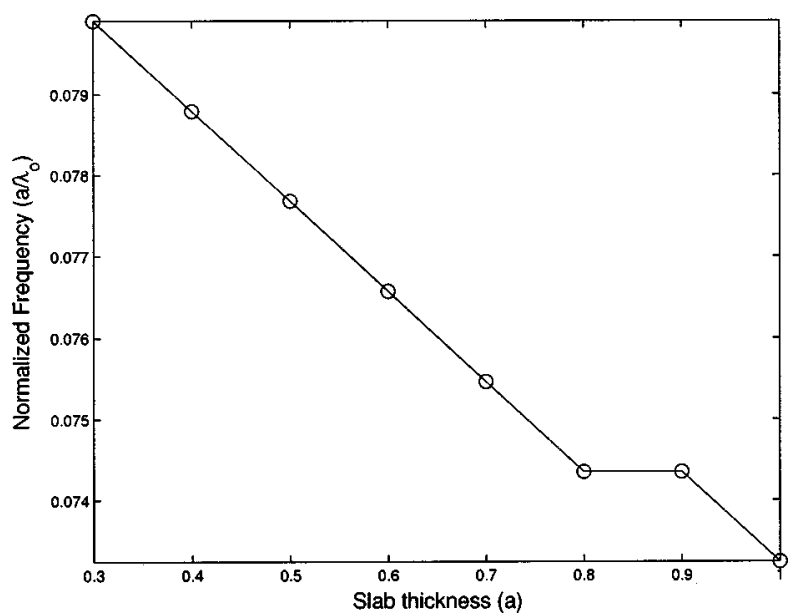

Fig. 8. Plot of the bandgap between the fundamental guided air band and dielectric band versus slab thickness.

the slab thickness is reduced, owing to increasing photon momentum in the $\hat{z}$ direction. However, the increase of the bandgap width with thinner dielectric slabs ${ }^{38}$ seems at first to be counterintuitive. A simple argument might be made to explain this result by analogy with the frequency dispersion relation for a photon in a constant index material,

$$
\left(\frac{\omega}{c}\right)^{2}=\frac{1}{n^{2}}\left(k_{\|}^{2}+k_{\perp}^{2}\right),
$$

where $n$ is the material refractive index, $c$ is the speed of light in vacuum, $k_{\perp}$ is the photon momentum normal to the slab, and $k_{\|}$is the in-plane momentum (in this discussion fixed at the $\mathrm{X}$ or $\mathrm{J}$ point). The bandgap increase results from the concentration of the air band mode in the air holes and the dielectric band in the high dielectric region. The energy required for a given photon momentum is larger in a lower-index material. Thus, as the slab becomes thinner and $k_{\perp}$ increases, the air band frequency increases faster than the dielectric band, and the bandgap increases. The variation of bandgap with slab thickness will have nonnegligible effects on the defect cavities analyzed below.

\section{THREE-DIMENSIONAL FINITE- DIFFERENCE TIME-DOMAIN ANALYSIS OF DEFECT MODES}

We now introduce a defect into the photonic crystal and analyze the properties of the localized modes that develop. By analyzing the defect modes in three dimensions, we are able to evaluate the effectiveness of the vertical confinement of the defect modes for a finite-thickness dielectric slab. The FDTD calculations in this section are performed for three different thicknesses of the slab $d$ : (i) $d=0.933 a$, (ii) $d=0.533 a$, and (iii) $d=0.4 a$. For frequencies in the bandgap of the photonic crystal, these thicknesses correspond to approximately $\lambda, \lambda / 2$, and $3 \lambda / 8$, where $\lambda$ is the wavelength of light in the slab.

To simulate the defect cavity we use the FDTD method, where we truncate the computational mesh by placing a nonreflecting absorber at all boundaries. ${ }^{39,40}$ Our computational mesh has a resolution of 15 points per interhole spacing, or equivalently 15 points per wavelength for frequencies within the photonic crystal bandgap. As in the previous calculations the radius of the air holes are equal to $0.3 a$. The number of layers of air holes that surround the defect is three, as shown in Fig. 3.

An initial TE-polarized electric field is used to excite the TE-like modes of the defect structure. Then the initial field is evolved in time with the FDTD method. A fast Fourier transform is applied to the resulting time series of the field at a point of low symmetry in the cavity, to pick out the resonance peaks of the defect mode. The field is then convolved in time with a bandpass filter to select out the defect modes. ${ }^{41}$ In the following calculation the radius of the defect hole is kept constant at $0.3 a$, whereas its refractive index is varied between 1.4 and 3.4. With the chosen $r / a$ and $n_{\text {slab }}$, and for the defect refractive-index range $1.4-3.4$, only one defect band is formed. A plot of the defect band frequency versus defect refractive index is given in Fig. 9 for the three different slab thicknesses.

Because we chose to use a circular defect, the symmetry of the defect cavity is the point group of the hexagonal

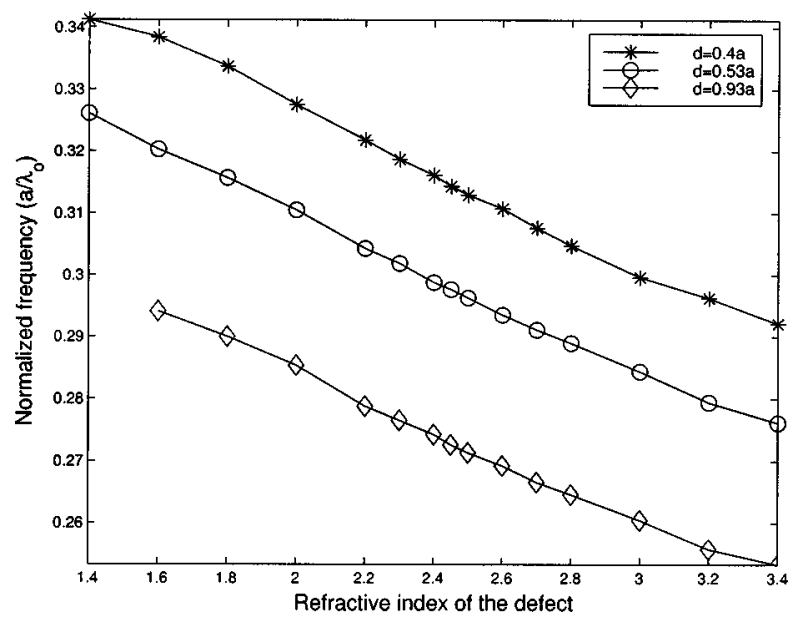

Fig. 9. Plot of the normalized frequency versus defect refractive index of the degenerate defect mode. The radius of the defect hole was kept constant at $0.3 a$, whereas the refractive index was varied. 
lattice of air holes $C_{6 v}$. The modes can be labeled by the irreducible representations (IRREP) of $C_{6 v} \cdot{ }^{42}$ We plotted the magnitude of the electric field of one of the modes of the defect band in Fig. 10(a). The defect mode transforms like the $x$ component of a vector under the pointgroup operations of $C_{6 v}$. Thus we label it an $x$ dipole mode. The character table of $C_{6 v}$ (Ref. 43) shows that the $x$ dipole mode is a member of a doubly degenerate IRREP. This IRREP can be represented by a pair of basis functions that transform like $x$ and $y$ vector components. We plotted the other defect mode, which we term the $y$ dipole mode, in Fig. 10(b). To excite the $x$ dipole mode we used an initial field with $\hat{x}$ symmetry. Similarly, for the $y$ dipole mode we used an initial field with $\hat{y}$ symmetry. The dipole modes are mainly TE polarized in the slab and have an antinode in the center of the defect region. This is important for maximizing the overlap of the defect mode and the semiconductor gain region, where we anticipate that electron and hole densities will be much lower

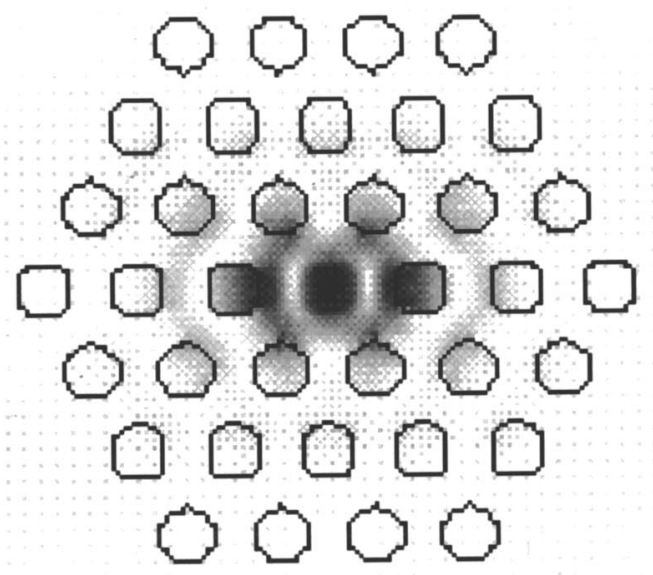

(a)

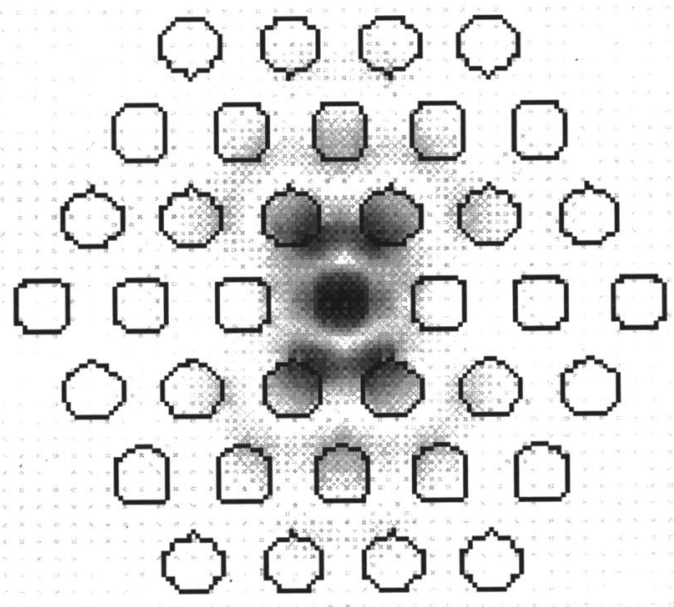

(b)

Fig. 10. 2D slice through the middle of the slab, showing the electric-field amplitudes of the degenerate defect modes. (a) $x$ dipole mode, (b) $y$ dipole mode.

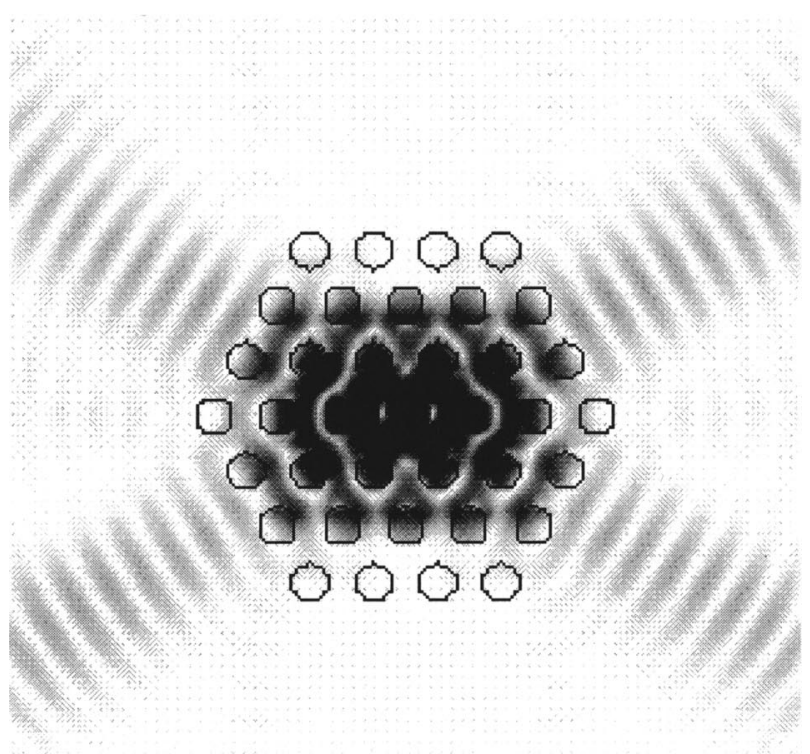

(a)

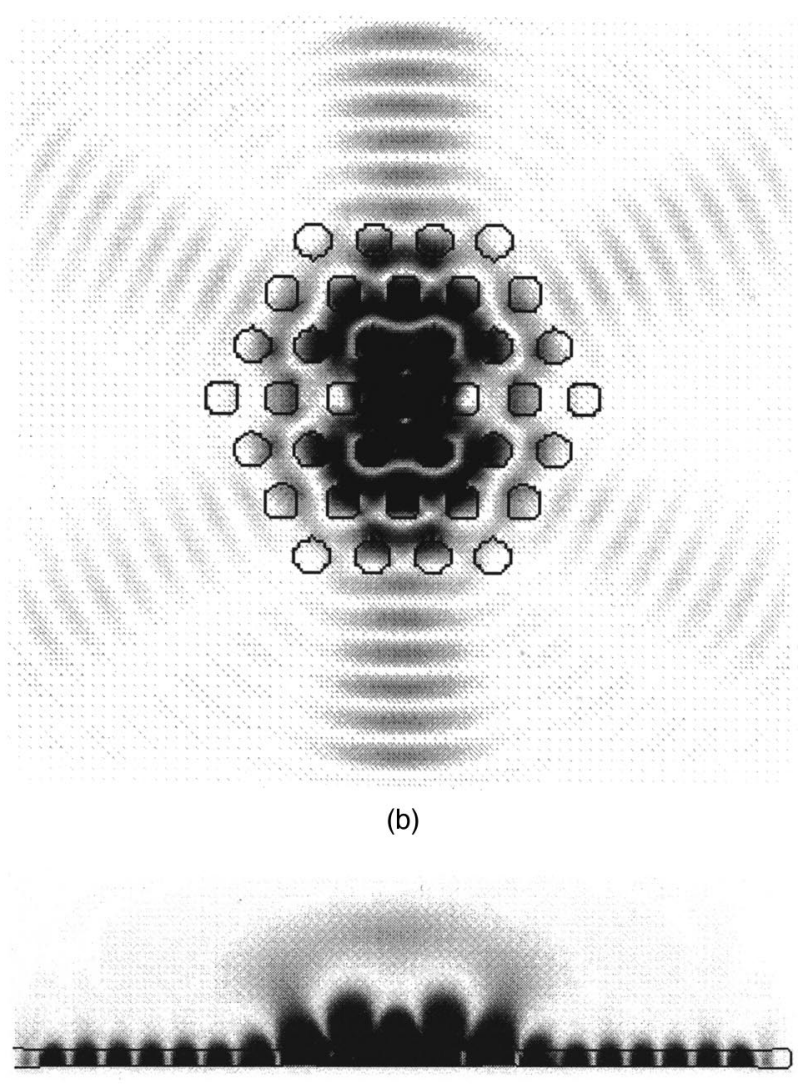

(c)

Fig. 11. Guided in-plane radiation losses of the $x$ and $y$ dipole modes (degenerate case) are shown in (a) and (b), respectively. In (c) a cross section along the $\hat{y}$ direction shows the radiation out the top half of the cavity for the $y$ dipole mode. In each plot the electric-field amplitude has been enhanced to highlight the losses.

near the surfaces of the etched holes. In Fig. 11 we show plots of the magnitude of the electric field for the $x$ and $y$ dipole modes, enhanced so as to highlight the radiation 
pattern in the in-plane directions. From the field plots one can see that the guided in-plane radiation is highly directional and is well suited for coupling to waveguides for in-plane routing. The fundamental $k$ component of the envelope of the defect modes is clearly near the $X$ point of the Brillouin zone. As discussed above, this is because the air band edge is at the $X$ point. Also plotted in Fig. 11 is the radiation pattern in the vertical direction for the $y$ dipole mode. With a definition of modal volume similar to that of Foresi et al. ${ }^{16}$ the volume of the $x$ and $y$ dipole modes is calculated to be approximately $2(\lambda / 2 n)^{3}$.

The in-plane symmetry of the defect modes allows us to apply the appropriate mirror conditions to reduce the computation size by one fourth. Because we are interested only in TE-like modes, we can also reduce the mesh size by a factor of 2 in the $\hat{z}$ direction by applying an even mirror boundary condition at the middle of the slab. Using the reduced mesh, we calculated the quality factor of the $y$ dipole mode for the defect refractive-index range 1.4-3.4. As the defect refractive index is varied, the dipole mode frequency sweeps across the bandgap as shown in Fig. 9; thus we are able to obtain plots of the quality factor versus frequency.

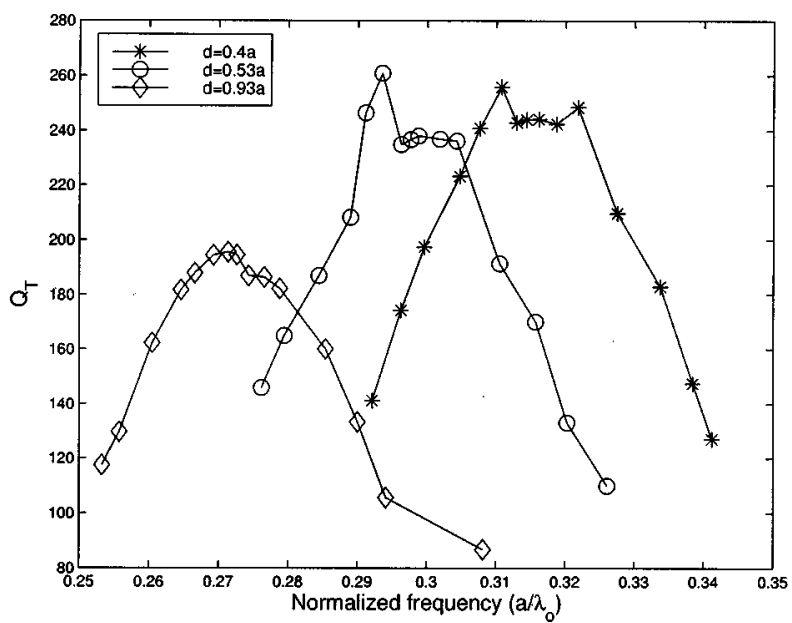

Fig. 12. Plot of the quality factor versus normalized frequency of the $y$ dipole mode.

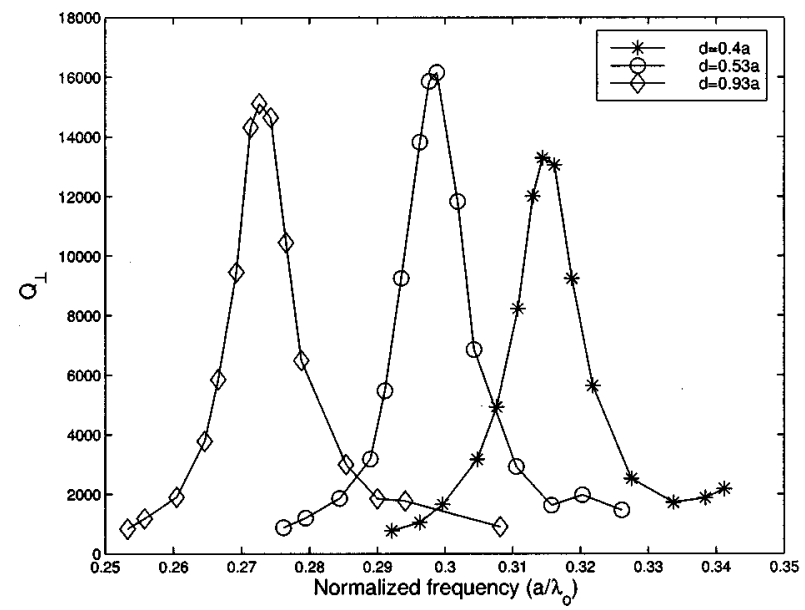

Fig. 13. Plot of the effective quality factor in the vertical direction $Q_{\perp}$ versus normalized frequency.

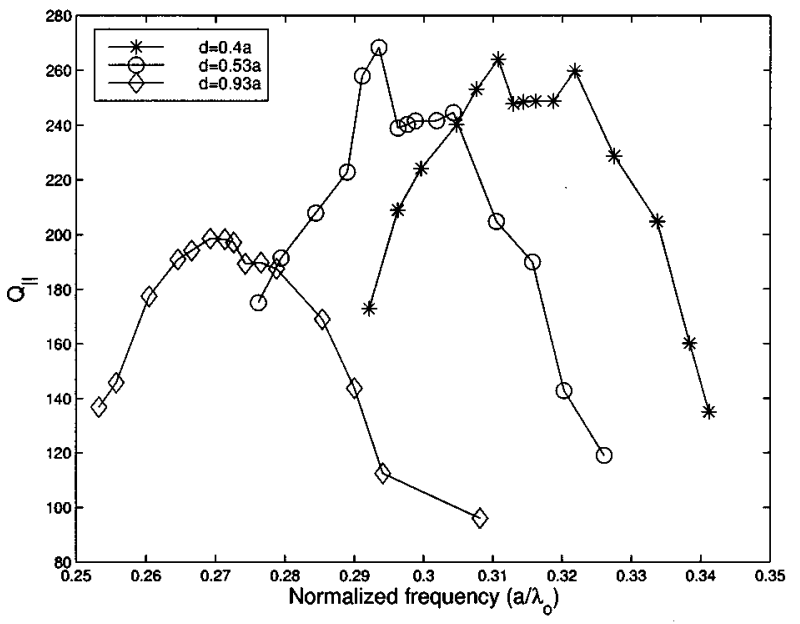

Fig. 14. Plot of the effective in-plane quality factor $Q_{\|}$versus normalized frequency.

We used two different methods to calculate the quality factor of the cavity modes. The first method measures the slope of the exponential decay of the energy of a given cavity mode ${ }^{44}$ :

$$
U(t)=U(0) \exp \left(-t / \tau_{\mathrm{ph}}\right)=U(0) \exp \left[-\left(\omega_{o} t\right) / Q\right],
$$

where $U$ is the energy in the mode and we have used the relation

$$
Q \equiv \omega_{o} \tau_{\mathrm{ph}} .
$$

The photon lifetime of the cavity mode is $\tau_{\mathrm{ph}}$, and $\omega_{o}$ is the angular frequency. This method is useful for relatively low $Q$ modes; however, for high $Q$ modes, the slope is nearly zero and an alternate method is employed. The second method calculates the power absorbed in the boundary $P(t)$ and divides it by the energy stored in the cavity mode, ${ }^{44}$

$$
Q \equiv \frac{\omega_{o} U(t)}{P(t)} .
$$

For the defect modes of the structure analyzed in this section we found little discrepancy between the two methods. In the following plots we chose to display the quality factor of the defect modes calculated with the second method, because this method allows one to separate out the radiation losses into different directions.

A plot of $Q$ versus normalized frequency is given in Fig. 12 for three different slab thicknesses: $d=0.933 a, d$ $=0.533 a$, and $d=0.4 a$. The quality factors of the defect modes seem at first sight to be rather unspectacular and the cavity losses too high to support lasing in such a small volume structure. To separate out radiation loss in the vertical direction that is due to leaky modes, and the losses that are due to the small number of photonic crystal layers that surround the defect region, we plot the effective vertical $Q$ and in-plane $Q$ for the $y$ dipole mode in Figs. 13 and 14, respectively. We define these effective quality factors as follows: 


$$
\begin{aligned}
\frac{\text { total power lost to boundary }}{\text { total energy stored in field }} & =\frac{P_{T}(t)}{U(t)} \\
& =\omega_{o}\left(\frac{1}{Q_{T}}\right) \\
& =\frac{P_{\perp}(t)+P_{\|}(t)}{U(t)} \\
& =\omega_{o}\left(\frac{1}{Q_{\perp}}+\frac{1}{Q_{\|}}\right) .
\end{aligned}
$$

$Q_{T}$ represents the total modal quality factor, whereas $Q_{\perp}$ is equal to the effective vertical quality factor, and $Q_{\|}$is equal to the effective in-plane quality factor. These effective $Q$ values are calculated by spatial separation of the power radiated by the dipole mode that is absorbed in the outer boundary. The in-plane radiation $P_{\|}$is defined as the radiated power that is absorbed into the sidewalls that extend from approximately a half-wavelength above the waveguide to a half-wavelength below the waveguide. The vertical radiation $P_{\perp}$ is then taken to be the absorbed power over the rest of the boundary. These effective $Q$ values, although not a strict separation of guided and leaky mode losses, do help to determine what factors are limiting the $Q$ of the defect modes.

A number of interesting features found in the plots of $Q$ are worth noting. As shown in Fig. 12, the total $Q$ drops off as the defect mode frequency approaches either the dielectric or the air bands, because the envelope function will decay into the photonic crystal more slowly. The relative heights of the peak effective in-plane quality factors for the different slab thicknesses is a result of the shrinking bandgap as described in Subsection 3.B. The thinnest slab, $d=0.4 a$, thus has the largest effective inplane $Q$. The strange structure near the peak of $Q_{\|}$disappears for more layers of photonic crystal that surround the defect ${ }^{45}$ and is most likely due to the waveguide mismatch between the patterned photonic crystal and the unpatterned waveguide at the exterior of the photonic crystal.

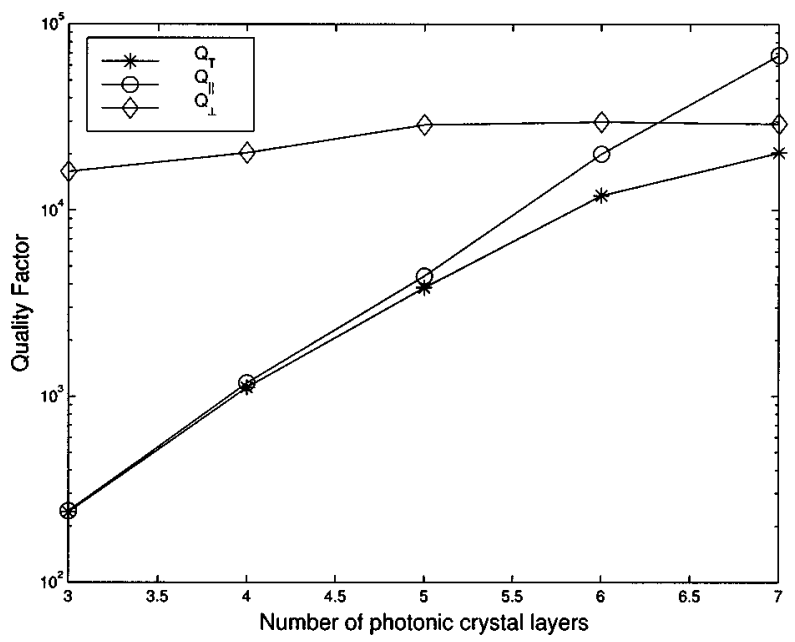

Fig. 15. Plot of the quality factor for increasing number of photonic crystal layers that surround the defect region.
The effective vertical $Q$ is a strong function of the position of the air band below the light line. This results as the defect modes are pulled out of the air band and into the frequency bandgap of the surrounding photonic crystal $^{4}$ as the dielectric constant of the defect is increased. Initially, as the dielectric constant of the defect is increased slowly above that of air, the defect modes are composed predominately of Bloch modes of the air band near the band edge (X point). The defect mode decays exponentially into the surrounding photonic crystal, owing to the forbidden energy gap there. This exponential decay is formed from a linear combination of $k$ components of the air band that surrounds the $\mathrm{X}$ point. Following the air band from the $\mathrm{X}$ point toward the $\Gamma$ point in Fig. 4, one crosses above the light line and the mode becomes leaky. It is this spread of $k$ components of the air band into the region above the light line that results in vertical losses of the defect cavity and a reduction of the $Q$ of the defect mode. The air band in Fig. 6 is well below the light line at the $\mathrm{X}$ and $\mathrm{J}$ points of the Brillouin zone; thus the defect modes couple only weakly to the leaky modes. From Figs. 9 and 13 the effective vertical $Q$ peaks sharply at a frequency just below the center of the bandgap for each slab thickness. The maximum $Q_{\perp}$ is approximately 16,000 for the slab of thickness $0.533 a$, well above the inplane $Q$, which is limited to 250 , owing to the small number of photonic crystal layers. The modal loss in this case is dominated by in-plane radiation, and the microcavity is essentially an in-plane emitter.

One can dramatically increase the total $Q$ of the defect modes and in the process change the device into a surface emitter by adding more photonic crystal layers. In Fig. 15 we plot the quality factor of the $y$ dipole mode for increasing numbers of layers of air holes that surround the defect $(d=0.533 a)$. As one would expect, $Q_{\|}$increases exponentially with the number of photonic crystal layers. $Q_{\perp}$ stays relatively constant, because adding more crystal layers is ineffective in capturing radiation in the vertical direction. The total $Q$ for three layers of photonic crystal is dominated by the in-plane losses, but as the number of layers increases, the total $Q$ asymptotically approaches $Q_{\perp}$. When the number of layers of holes surrounding the defect is changed from 3 to $7, Q_{\|}$can theoretically be increased well above $Q_{\perp}$, thus resulting in a vertical emitter with a total $Q$ near 20,000. Of course, realistically the quality of the fabrication of the $2 \mathrm{D}$ photonic crystal will limit the obtainable in-plane $Q$. The optical cavity can also be designed to radiate in the vertical direction by simple adjustment of the $r / a$ ratio so as to shift the air band frequency closer to light line, thereby increasing the defect mode coupling to leaky modes.

\section{DEGENERACY SPLITTING OF DIPOLE MODE}

In the defect cavity analyzed in Section 4 we found that the localized defect modes were a pair of doubly degenerate dipole modes. Here we analyze the effects of creating a defect that is of lower symmetry than the hexagonal lattice, thereby splitting the dipole degeneracy. Because the $x$ dipole mode is extended in the $\hat{x}$ direction, one method of splitting the degeneracy would be to increase 


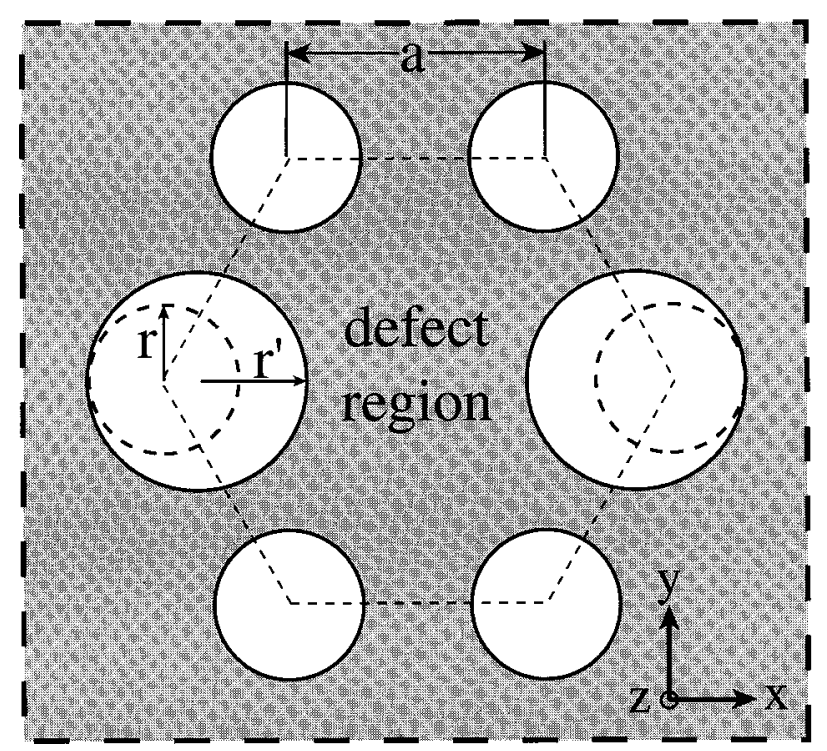

Fig. 16. Cavity geometry for splitting of the dipole mode degeneracy. Only the nearest neighbor holes of the defect are shown. The two nearest holes in the $x$ direction are enlarged. The central defect hole is filled in and has a dielectric constant equal to that of the slab. Notice that the two enlarged holes are also moved inward toward the central hole to preserve the spacing between holes in the $\hat{x}$ direction.

the nearest neighbor holes along the $\hat{x}$ axis. This has the effect of lowering the symmetry of the defect cavity to $C_{2 v}$, which splits the $(x, y)$ IRREP of $C_{6 v}$ into two nondegenerate IRREP's, thus splitting the $x$ and $y$ dipole modes.

An illustration of the modified cavity is given in Fig. 16. The 3D FDTD calculations are done only for a slab thickness of $0.533 a$. The radius of all holes, except those we modified explicitly, have $r / a=0.3$. The refractive index of the defect hole is set to $n_{\text {defect }}=3.4$, which corresponds to removal of a single hole. Now, instead of varying the defect hole index, the hole radius $r^{\prime}$ of the two nearest neighbor holes along the $\hat{x}$ axis is varied, as shown on Fig. 16. Also, as $r^{\prime}$ is increased the two holes are moved simultaneously toward the center defect so as to maintain the rib size in the $\hat{x}$ direction.

A plot of $Q, Q_{\perp}$, and $Q_{\|}$versus $r^{\prime} / a$ is given in Fig. 17 for the $y$ dipole mode. The $Q$ factors peak for a dipole mode frequency just below the center of the bandgap. The peak value of $Q_{\perp}$ is lower than in the degenerate structure, but it is still relatively large. A Fourier spectrum of the time-evolved field for the structure with $r^{\prime}$ $=0.35 a$ is shown in Fig. 18. The $x$ dipole is shifted much higher in frequency than the $y$ dipole, because the $x$ dipole has more of an overlap with the enlarged air holes. At larger $r^{\prime} / a$ ratios the $x$ dipole mode is pushed completely out of the bandgap, whereas the $y$ dipole frequency is shifted only slightly. The frequency separation between the $x$ and $y$ dipoles increases above 0.04 (in normalized units) for $r^{\prime} / a>0.4$. This corresponds to a wavelength separation of approximately $200 \mathrm{~nm}$, assuming the $y$ dipole is resonant at a wavelength of $1.55 \mu \mathrm{m}$. The linewidth of the spontaneous emission from quantum wells at this wavelength is approximately 200 -nm wide ${ }^{13}$; thus the mode splitting forces the $x$ dipole out of the inhomogeneously broadened emission linewidth, creating a

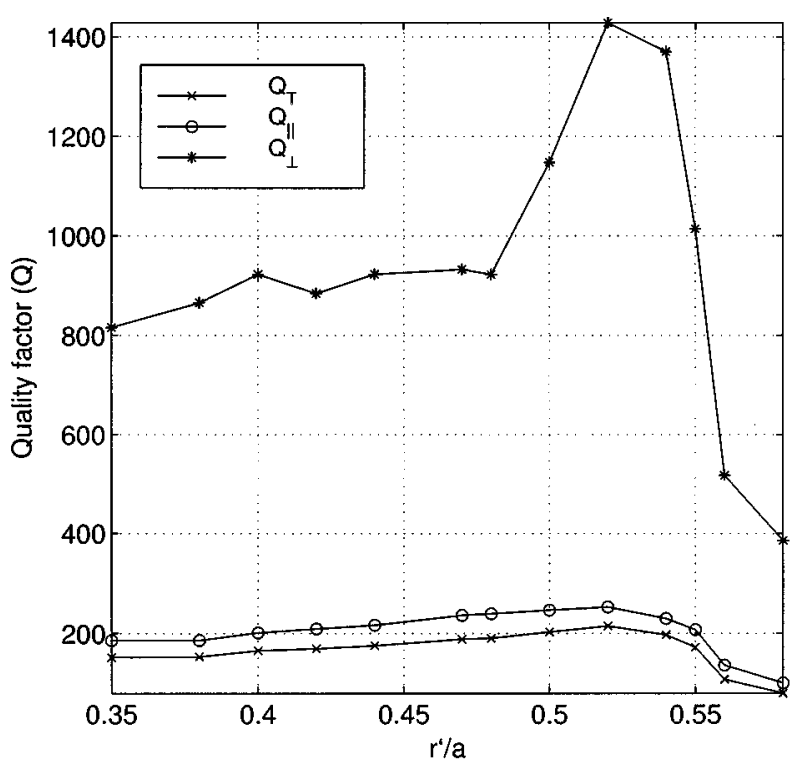

(a)

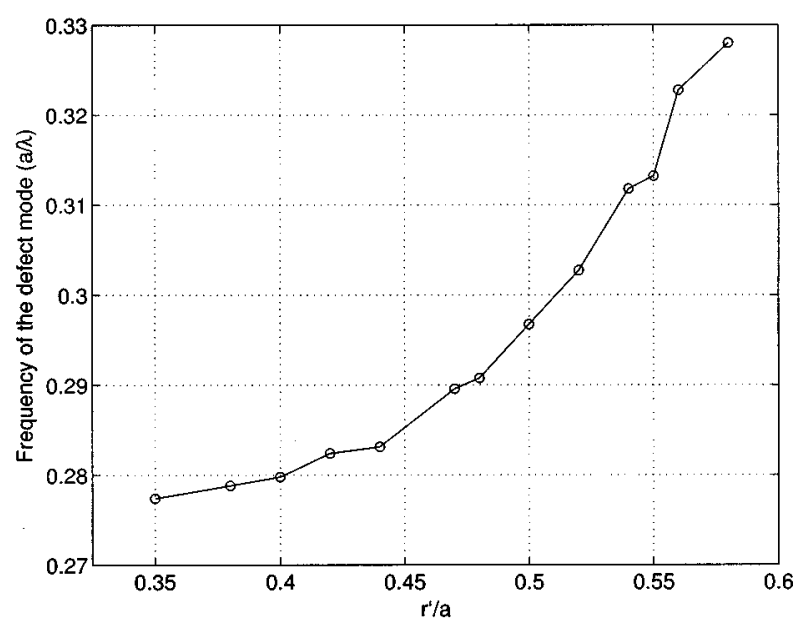

(b)

Fig. 17. (a) Plot of the calculated $Q$ of the $y$ dipole as a function of $r^{\prime} / a$. (b) Plot of the normalized frequency of the $y$ dipole mode.

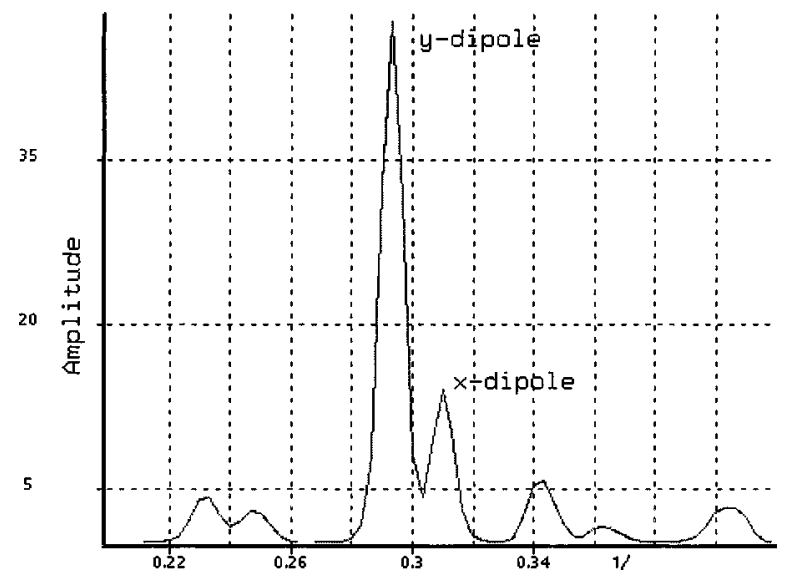

Fig. 18. Fourier spectrum of an initial field chosen to excite both the $x$ and $y$ dipole modes. $r^{\prime} / a=0.35$ in this case. For larger $r^{\prime} / a$ ratios the splitting of the $x$ and $y$ dipoles is strong enough to push the $x$ dipole frequency out of the bandgap. The $y$ dipole, however, is still highly localized, and its frequency is changed only moderately. 
cavity with a single high- $Q$ mode. Of course, because the microcavity is formed from a photonic crystal with only an in-plane photonic bandgap as opposed to a full 3D photonic bandgap structure, there are still radiation modes that couple to the quantum well active regions.

\section{CONCLUSION}

We have analyzed the defect modes of a $2 \mathrm{D}$ photonic crystal microcavity in a thin slab of high-index material, using a three-dimensional (3D) finite-difference timedomain (FDTD) method. We find that the high-Q, localized defect modes of the $2 \mathrm{D}$ photonic crystal with a single missing hole are a pair of degenerate $(x, y)$ dipole modes. The quality factor of the cavity modes are calculated for varying membrane thicknesses. The defect mode can be either a surface emitter or an in-plane emitter, depending on the number of layers of $2 \mathrm{D}$ photonic crystal that surround the defect. A maximum $Q$ for the slab thickness of $0.5 a$ is calculated theoretically to be as great as 20,000, limited by radiative losses in the vertical direction. We have also analyzed a lower-symmetry defect cavity, where the $x$ and $y$ dipole mode degeneracy is split, thus creating a truly single high- $Q$ mode cavity.

\section{ACKNOWLEDGMENTS}

The authors thank B. D'Urso for his helpful suggestions with the FDTD calculations. O. Painter acknowledges support from the Natural Sciences and Engineering Research Council of Canada. This study was supported by the U.S. Army Research Office under contract DAAH0496-1-0389 and the National Science Foundation under contract ECS-9632937.

O. Painter's e-mail address is opainter@cco.caltech.edu.

\section{REFERENCES AND NOTES}

1. E. M. Purcell, "Spontaneous emission probabilities at radio frequencies," Phys. Rev. 69, 681 (1946).

2. D. Kleppner, "Inhibited spontaneous emission," Phys. Rev. Lett. 47, 233-236 (1981).

3. E. Yablonovitch, "Inhibited spontaneous emission in solidstate physics and electronics," Phys. Rev. Lett. 58, 2059 (1987).

4. J. D. Joannopoulos, R. D. Meade, and J. N. Winn, Photonic Crystals (Princeton U. Press, Princeton, N.J., 1995).

5. S. L. McCall, P. M. Platzman, R. Dalichaouch, D. Smith, and S. Schultz, "Microwave propagation in two-dimensional dielectric lattices," Phys. Rev. Lett. 67, 2017-2020 (1991).

6. E. Yablonovitch, T. J. Gmitter, R. D. Meade, A. M. Rappe, K. D. Brommer, and J. D. Joannopoulos, "Donor and acceptor modes in photonic band-structure," Phys. Rev. Lett. 67, 3380-3383 (1991).

7. B. P. V. der Gaag and A. Scherer, Appl. Phys. Lett. 56, 481483 (1989).

8. C. C. Cheng, A. Scherer, V. Arbet-Engels, and E. Yablonovitch, "Lithographic band gap tuning in photonic band gap crystals," J. Vac. Sci. Technol. B 14, 4110-4119 (1996).

9. T. Krauss, Y. P. Song, S. Thoms, C. D. W. Wilkinson, and R. M. D. L. Rue, "Fabrication of 2-D photonic bandgap structures in GaAs/AlGaAs," Electron. Lett. 30, 1444-1446 (1994).
10. G. Feiertag, W. Ehrfeld, H. Freimuth, H. Kolle, H. Lehr, M Schmidt, M. M. Sigalas, C. M. Soukoulis, G. Kiriakidis, T. Pederson, J. Kuhl, and W. Koenig, "Fabrication of photonic crystals by deep x-ray lithography," Appl. Phys. Lett. 71, 1441-1443 (1997)

11. K. Inoue, M. Wada, K. Sakoda, M. Hayashi, T. Fukushima, and A. Yamanaka, "Near-infrared photonic band gap of two-dimensional triangular air-rod lattices as revealed by transmittance measurement," Phys. Rev. B 53, 1010-1013 (1996).

12. J. L. Jewell, J. P. Harbison, A. Scherer, Y. H. Lee, and L. T. Florez, "Vertical-cavity surface-emitting lasers: design, growth, fabrication, characterization," IEEE J. Quantum Electron. QE-27, 1332-1346 (1996).

13. A. F. J. Levi, S. L. McCall, S. J. Pearton, and R. A. Logan, "Room temperature operation of submicrometre radius disk laser," Electron. Lett. 29, 1666-1667 (1993).

14. S. L. McCall, A. F. J. Levi, R. E. Slusher, S. J. Pearton, and R. A. Logan, "Whispering-gallery mode lasers," Appl. Phys. Lett. 60, 289-291 (1992).

15. D. Y. Chu and S.-T. Ho, "Spontaneous emission from excitons in cylindrical dielectric waveguides and the spontaneous-emission factor of microcavity ring lasers," J. Opt. Soc. Am. B 10, 381-390 (1993).

16. J. S. Foresi, P. R. Villeneuve, J. Ferrera, E. R. Thoen, G. Steinmeyer, S. Fan, J. D. Joannopoulos, L. C. Kimerling, H I. Smith, and E. P. Ippen, "Photonic-bandgap microcavities in optical waveguides," Nature (London) 390, 143-145 (1997).

17. J. P. Zhang, D. Y. Chu, S. L. Wu, W. G. Bi, R. C. Tiberio, R. M. Joseph, A. Taflove, C. W. Tu, and S. T. Ho, "Nanofabrication of 1-D photonic bandgap structures along a photonic wire," IEEE Photonics Technol. Lett. 8, 491-493 (1996).

18. T. F. Krauss, B. Vögele, C. R. Stanley, and R. M. D. L. Rue, "Waveguide microcavity based on photonic microstructures," IEEE Photonics Technol. Lett. 9, 176-178 (1997).

19. T. F. Krauss, R. M. D. L. Rue, and S. Brand, "Twodimensional photonic-bandgap structures operating at near-infrared wavelengths," Nature (London) 383, 699-702 (1996).

20. J. O'Brien, O. Painter, C. C. Cheng, R. Lee, A. Scherer, and A. Yariv, "Lasers incorporating 2D photonic bandgap mirrors," Electron. Lett. 32, 2243-2244 (1996).

21. T. Baba and T. Matsuzaki, "Fabrication and photoluminescence of GaInAsP/InP 2D photonic crystals," Jpn. J. Appl. Phys., Part 2 35, 1348-1352 (1996).

22. T. Hamano, H. Hirayama, and Y. Aoyyagi, "Optical characterization of GaAs 2D photonic bandgap crystal fabricated by selective MOVPE," in Conference on Lasers and ElectroOptics, Vol. 11 of 1997 OSA Technical Digest Series (Optical Society of America, Washington, D.C., 1997), pp. 528529.

23. T. Baba, "Photonic crystals and microdisk cavities based on GaInAsP-InP system," IEEE J. Sel. Topics Quantum Electron. 3, 808-830 (1997).

24. P. R. Villeneuve, S. Fan, and J. D. Joannopoulos, "Microcavities in photonic crystals: mode symmetry, tunability, and coupling efficiency," Phys. Rev. B 54, 7837-7842 (1996).

25. H. Yokoyama, "Physics and device application of optical microcavities," Science 256, 66-70 (1992).

26. I. Schnitzer, E. Yablonovitch, A. Scherer, and T. J. Gmitter, in Photonic Band Gaps and Localization (Kluwer Academic, Dordrecht, The Netherlands, 1996), pp. 369-378.

27. K. S. Yee, "Numerical solution of boundary value problems involving Maxwell's equations in isotropic media," IEEE Trans. Antennas Propag. 14, 302-307 (1966).

28. G. Björk and Y. Yamamoto, "Analysis of semiconductor microcavity lasers using rate equations," IEEE J. Quantum Electron. QE-27, 2386-2396 (1991).

29. D. J. Heinzen, J. J. Childs, J. E. Thomas, and M. S. Feld, "Enhanced and inhibited visible spontaneous emission by atoms in a confocal resonator," Phys. Rev. Lett. 58, 1320 1323 (1987).

30. L. A. Coldren and S. W. Corzine, Diode Lasers and Photonic Integrated Circuits (Wiley, New York, 1995). 
31. O. Painter, R. Lee, A. Yariv, A. Scherer, and J. O’Brien, "Photonic bandgap membrane microresonator," in Integrated Photonics Research, Vol. 4 of 1998 OSA Technical Digest Series (Optical Society of America, Washington, D.C., 1998), pp. 221-223.

32. Y. Zou, J. S. Osinski, P. Grodzinski, P. D. Dapkus, W. Rideout, W. F. Sharfim, and F. D. Crawford, "Experimental verification of strain benefits in $1.5 \mu \mathrm{m}$ semiconductor lasers by carrier lifetime and gain measurements," IEEE Photonics Technol. Lett. 4, 1315-1318 (1992).

33. S. Adachi, "Material parameters of $\operatorname{In}_{1-x} \mathrm{Ga}_{x} \mathrm{As}_{y} \mathrm{P}_{1-y}$ and related binaries," J. Appl. Phys. 53, 8775-8792 (1982).

34. M. Plihal and A. A. Maradudin, "Photonic band structure of two-dimensional systems: the triangular lattice," Phys. Rev. B 44, 8565-8571 (1991).

35. D. M. Atkin, P. S. J. Russell, T. A. Birks, and P. J. Roberts, "Photonic band structure of guided Bloch modes in high index films fully etched through with periodic microstructure," J. Mod. Opt. 43, 1035-1053 (1996).

36. C. T. Chan, Q. L. Yu, and K. M. Ho, "Order- $N$ spectral method for electromagnetic waves," Phys. Rev. B 51, 16,635-16,642 (1995).

37. G. Mur, "Absorbing boundary conditions for the finitedifference approximation of the time-domain electromagnetic-field equations," IEEE Trans. Electromagn. Compat. 23, 377-382 (1981).

38. P. S. J. Russell, D. M. Atkin, and T. A. Birks, in Microcavi- ties and Photonic Bandgaps (Kluwer Academic, Dordrecht, The Netherlands, 1996), pp. 203-218.

39. K. Chamberlain and L. Gordon, "Modeling good conductors using the finite-difference, time-domain technique," IEEE Trans. Electromagn. Compat. 37, 210-216 (1995).

40. B. D'Urso, O. Painter, J. O’Brien, T. Tombrello, A. Scherer, and A. Yariv, "Modal reflectivity in finite-depth twodimensional photonic-crystal microcavities," J. Opt. Soc. Am. B 15, 1155-1159 (1998).

41. D. H. Choi and W. J. R. Hoefer, "The finite-difference-timedomain method and its application to eigenvalue problems," IEEE Trans. Microwave Theory Tech. 34, 1464-1469 (1986).

42. K. Sakoda, "Symmetry, degeneracy, and uncoupled modes in two-dimensional photonic crystals," Phys. Rev. B 52, 7982-7986 (1995).

43. M. Tinkham, Group Theory and Quantum Mechanics (McGraw-Hill, New York, 1964).

44. J. D. Jackson, Classical Electrodynamics (Wiley, New York, 1962).

45. O. Painter, J. Vučković, and A. Scherer, in an unpublished study, analyze similar microcavities, as presented in this paper, although with different bottom substrates of the waveguide and with increasing number of photonic crystal layers. Calculations of $Q_{T}, Q_{\|}$, and $Q_{\perp}$ versus frequency of the defect mode with seven layers of photonic crystal shows a smooth peak for all the $Q$ 's, with none of the structure present for only three layers. 